\title{
3. Recombination in Radar Meteors
}

RICHARD B. SoUTHWORTH

Smithsonian Astrophysical Observatory

Cambridge, Massachusetts

$\mathrm{R}$ ECOMBination OF THE ELECTRONS in the ionized column has often been considered (see, for example, Öpik, 1955), but the uncertainties in the atomic and molecular species present and in the rate constants have made it difficult to predict. Furthermore, recombination has not been recognized in the observations, so that it has been judged to be negligible (McKinley, 1961). More recently, however, a relatively high rate constant has been found for dissociative recombination of certain molecular ions. We have now observed recombination at comparable rates in our radar meteor recordings.

\section{RECOMBINATION RATE}

Recombination rates for all meteoric atoms are too slow to interest us here. Mehr and Biondi (1969) have obtained reliable laboratory measures for dissociative recombination of the most likely molecules in the meteor environment, $\mathrm{N}_{2}{ }^{+}$and $\mathrm{O}_{2}{ }^{+}$. We do not know what atoms or molecules are first ionized in the meteoric process. However, if metallic ions, such as are seen in meteor spectra, are created first, we may plausibly expect fast charge transfer to the atmospheric molecules (Öpik, 1958). Then, recombination can be expressed by

Mehr and Biondi (1969) found

$$
\frac{d n}{d t}=-\alpha n n_{i}
$$

where $n$ is the electron volume density in $\mathrm{cm}^{-3}$, and $n_{i}$ is the volume density of ion species that recombine at rate $\alpha \mathrm{cm}^{3} \mathrm{~s}^{-1}$.

$$
\begin{aligned}
& \alpha\left(\mathrm{O}_{2}{ }^{+}\right)=1.9 \times 10^{-7}\left(\frac{T_{e}}{300}\right)^{-0.70}, \\
& 300^{\circ} \mathrm{K}<T_{e}<700^{\circ} \mathrm{K}
\end{aligned}
$$

$$
\begin{aligned}
& \alpha\left(\mathrm{N}_{2}{ }^{+}\right)=1.9 \times 10^{-7}\left(\frac{T_{e}}{300}\right)^{-0.39}, \\
& 300^{\circ} \mathrm{K}<T_{\epsilon}<4500^{\circ} \mathrm{K}
\end{aligned}
$$

where $T_{e}$ is the electron temperature, and the gas and ion temperatures were held at $300^{\circ} \mathrm{K}$. As a first approximation, one may take the ambient temperature $\sim 200^{\circ} \mathrm{K}$ for $T_{e}$. If all ions recombine at rate $\alpha$, we have $n_{i}=n$. Here, we will use

$$
\frac{d n}{d t}=-2 \times 10^{-7} n^{2}
$$

allowing for other ions that do not recombine so fast and for some atmospheric heating. The observations are not sensitive to an uncertainty in $\alpha$ of much less than a factor of 2 .

\section{ANALYTIC APPROXIMATION}

If we neglect the effect of recombination on the radial distribution of electrons in the column, we can compute the effect of recombination on the total line density on the electrons as follows. The density at distance $r$ from the column axis is taken to be gaussian with radius $\rho$ :

$$
n=\left(\frac{q}{\pi \rho^{2}}\right) \exp \left(\frac{-r^{2}}{\rho^{2}}\right)
$$

where $q$ is the electron line density. Initially, we have $q=q_{0}$ the original line density, and $\rho=r_{0}$ the initial radius. The effect of diffusion (neglecting recombination) is

$$
\rho^{2}=r_{0}{ }^{2}+4 D t
$$


and the effect of recombination is

$$
\frac{d q}{d t}=-2 \pi \alpha \int_{0}^{\infty} n^{2} r d r=-\frac{\alpha q^{2}}{2 \pi \rho^{2}}
$$

When we combine equations (6) and (7),

$$
\frac{d q}{q^{2}}=-\alpha \frac{d t}{2 \pi r_{0}^{2}+8 \pi D t}
$$

then, integrating with respect to time, we find

$$
\frac{1}{q}=\frac{1}{q_{0}}+\frac{1}{q_{1 \mathrm{im}}}
$$

where

$$
q_{\lim }=\frac{8 \pi D}{\alpha \ln \left[1+\left(4 D t / r_{0}^{2}\right)\right]}
$$

Qualitatively, the result of recombination is to reduce any initial line density to less than an upper bound $q_{\text {lin }}$.

The exponential decay of the radar signal with time, caused by diffusion, is often used to measure the meteor's height. Diffusion alone reduces the received signal voltage by the factor

$$
L=\exp \left[-\left(\frac{2 \pi}{\lambda}\right)^{2}\left(r_{0}{ }^{2}+4 D t\right)\right]
$$

(McKinley, 1961), where $\lambda$ is the radar wavelength (733.1 cm for our equipment). Differentiating (11), we have

$$
D=-\left(\frac{\lambda}{4 \pi}\right)^{2} \frac{d L}{L d t}
$$

which is used to find $D$ and thence height from the observed decay $d L / L d t$.

If there is recombination in addition to diffusion and if the recombination is not taken into account (to do so is difficult in practice), the height will be overestimated. The error in height can be estimated from equations (12) and (8) as

$\Delta h_{D}=H \ln \frac{D^{\prime}}{D}=H \ln \left[1+\frac{\lambda^{2} \alpha q}{32 \pi^{3} D\left(r_{0}^{2}+4 D t\right)}\right]$

where $H$ is the atmospheric scale height, and $D^{\prime}$ the incorrectly inferred value of $D$.

\section{EXACT INTEGRATIONS}

Numerical integrations were performed to find the actual distribution of electrons under com- bined recombination and diffusion. The electron density was computed at small intervals of space and time for $1 \mathrm{~s}$ after formation of the ionized column. The initial electron distribution was assumed to be gaussian, with radii approximating Manning's (1958) or Oppik's (1955) estimates, as tabulated by Southworth (1962).

Table 1 shows some results for a variety of values of diffusion $D\left(\mathrm{~cm}^{2} \mathrm{~s}^{-2}\right)$, initial radius $r_{0}(\mathrm{~cm})$, and initial line density $q_{0}\left(\mathrm{~cm}^{-1}\right)$. The tabulated cases cover the brighter end of the range of line densities observed with our equipment; fainter meteors are little affected by recombination. To fix ideas, diffusion has been translated into height $h_{d}(\mathrm{~km})$ by use of Greenhow and Neufeld's (1955) results as expressed by McKinley (1961):

$$
\log _{10} D=0.067 h_{D}-1.6
$$

line density has been translated into radar magnitude $M$ by Kaiser's (1955) relation

$$
M=35-2.5 \log _{10} q
$$

At each height, the smaller value of initial radius is approximately Manning's estimate, and the larger value is approximately Öpik's.

Results are tabulated for $0.03 \mathrm{~s}$ after formation of the ionized column; this time is representative for our observations of the electrons in the principal Fresnel zone. The accuracy of approximations (10) and (13) is similar at other times.

The tabulated values of $M_{\text {lim }}$ are deduced from $M_{0}$ and the accurately computed $M$ by equations (9) and (15); $\Delta M_{1 \mathrm{im}}$ is the difference $\left(M_{\text {lim }}^{\prime}-M_{\text {lim }}\right)$ between $M_{\text {lim }}$ and analytic approximation $M_{1 \text { im }}^{\prime}$ deduced by equations (10) and (15). One sees that the error in the analytic approximation is small for all tabulated cases, and one can infer that it is small for all meteors observed with our equipment.

The integrations also yield the rates of decay caused by combined diffusion and recombination. These have been converted into height differences $\Delta h_{D}$ by use of equation (14) and compared with predicted values from (13). The differences between approximation and integration are small for all cases computed.

The effect of recombination on the relative distribution of electrons in the column was carefully 
TABLE 1.-Results of Numerical Integrations Compared with Approximation

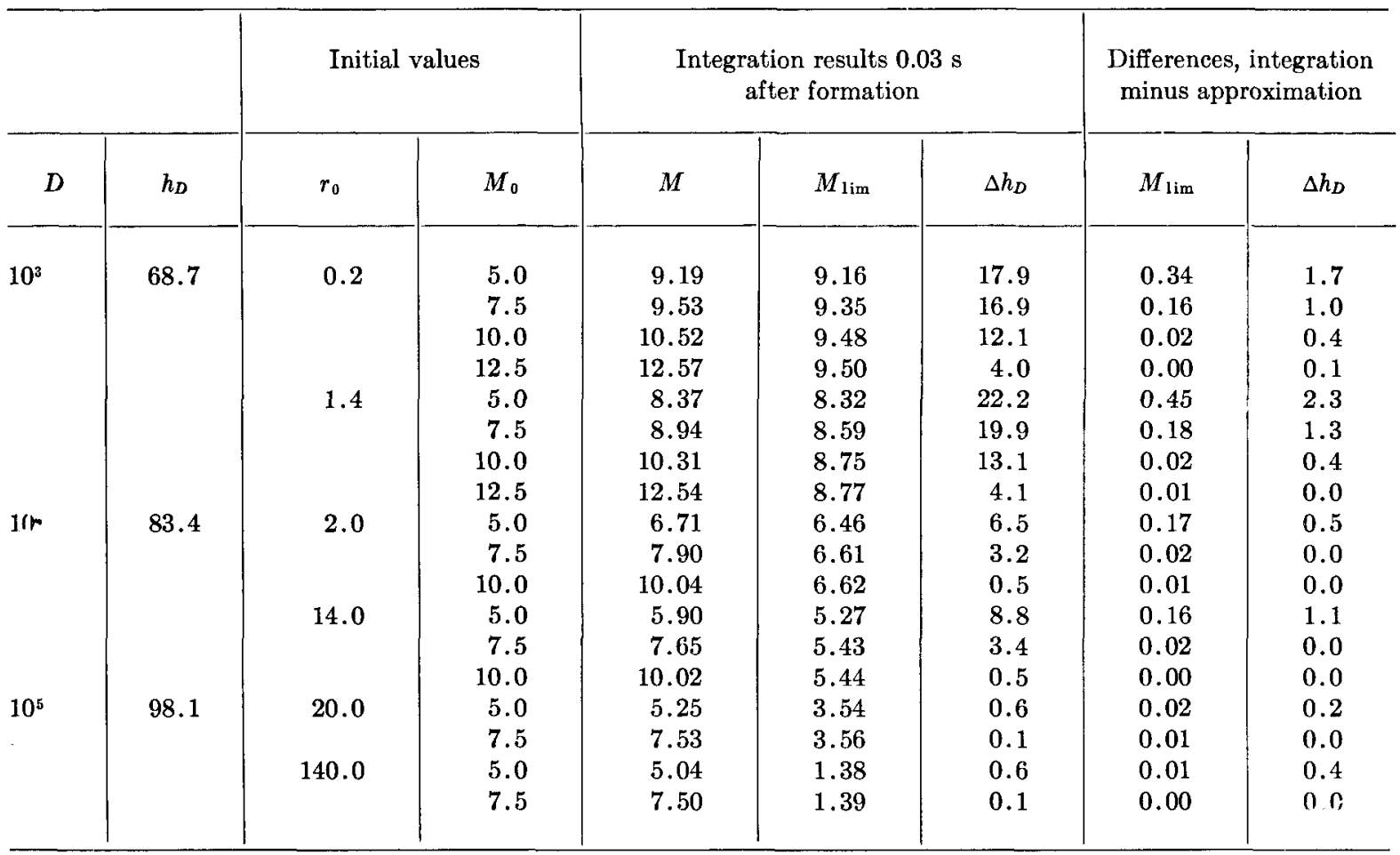

examined; it turned out to be small in all the tabulated cases. The gaussian distribution represented by equations (5) and (6) was always a good approximation. An apparent magnitude computed from (5) and (6) would always have been between 0.0 and 0.2 mag too bright.

\section{OBSERVED IONIZATION CURVES}

For each observed meteor, we compute an "ionization curve" showing the initial radar magnitude of the ionized column as a function of position along the column; the position is represented by the time that the meteoroid passed that position. Each of the maxima and minima (collectively "extrema") of the Fresnel pattern from each station yields one point on the ionization curve. The deviations from a smooth curve of the extrema after the first maximum measure the amount of ionization in the later Fresnel zones. The effective length of the $i$ th extremum $(i>1)$ is

$$
F_{i} \approx \frac{1}{\pi} \sqrt{\frac{\lambda R}{2[i-(3 / 4)]}}
$$

and is measured

$$
t_{i} \approx \frac{1}{4 V} \sqrt{\frac{\lambda R}{2[i-(3 / 4)]}}
$$

after the meteoroid passes the center of the zone. Here, $R \sim 150$ to $300 \mathrm{~km}$ is the distance to the ionized column, and $V$ is the meteor velocity.

We have observed recombination in three ways. We first saw it in ionization curves derived as just described. Figure 1 is an example. The digits represent radar magnitudes derived from the principal Fresnel zone (left-most digit) and later zones as observed at stations $2,3,4$, and 6 , all corrected for diffusion in the interval since the zone was formed. Recombination is seen in the upward slope of the first few digits for each station; these show that the electron line density appears greater when measured sooner after formation, even after correction for diffusion. (The later digits for each station show fragmentation.) It is not plausible that the ionization curve should have a bump just after the principal zone for each station, on this and on many other 


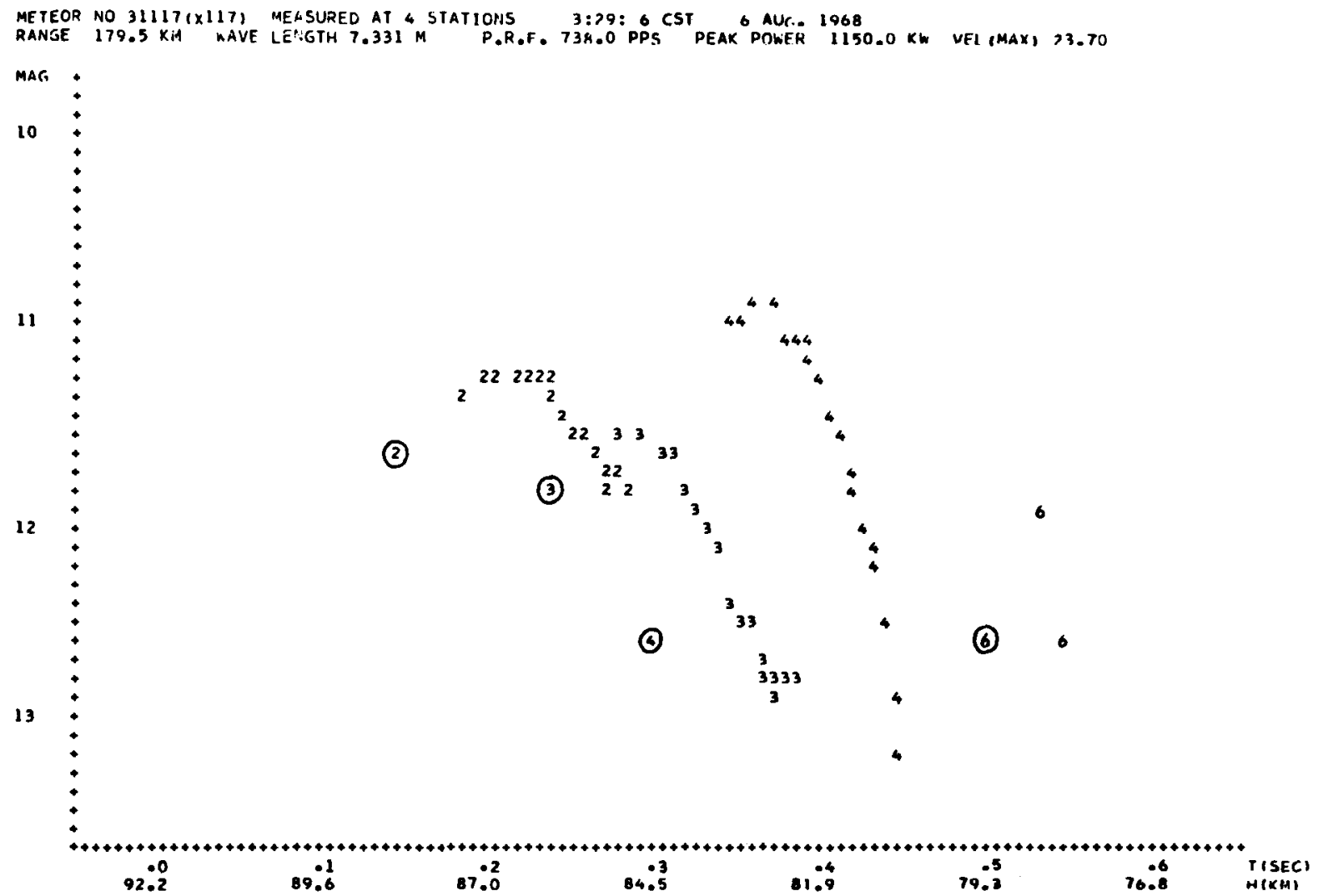

FIGURE 1.-An observed ionization curve, showing recombination and fragmentation. The circled digits represent values from the principal Fresnel zones for stations 2, 3, 4, and 6 . The following digits represent values from the later zones at each station.

similar meteors. Some process that removes electrons from the column for only a few hundredths of a second is required, and recombination suits.

\section{HEIGHT MAGNITUDE DISTRIBUTION}

Diffusion heights were computed from 13672 meteors observed in 1962 to 1965 by using Greenhow and Neufeld's (1955) profile (14). Their result was chosen because it is in harmony with atmospheric models, not because it seemed a good determination. (We believe that their statistical analysis is faulty because selection effects were ignored.) Greenhow and Hall's (1960) more careful diffusion measures are vitiated by recombination, as we discuss later. The effect of recombination on our diffusions is small for many of the relatively faint meteors that we observe.
We observe apparent diffusion at three to seven different points on each meteor trail and combine these values into a mean value of the diffusion height at the maximum of the ionization curve of each meteor. We also find an internal standard error of that height, the apparent atmospheric scale height, and its standard error. In the present analysis, we use the errors primarily to eliminate inconsistent and distorted data.

Figure 2 shows the distribution of diffusion height at the maximum of the ionization curve, as a function of the magnitude at the maximum, for 10163 meteors. Meteors whose standard error in height exceeded $4 \mathrm{~km}$ or that yield an unreasonable scale height have been omitted. True height will differ from these diffusion heights by up to about $3 \mathrm{~km}$, depending on height and probably also on time. True heights are not available for these meteors; but diffusion heights are preferable for the present purposes because we are examining 


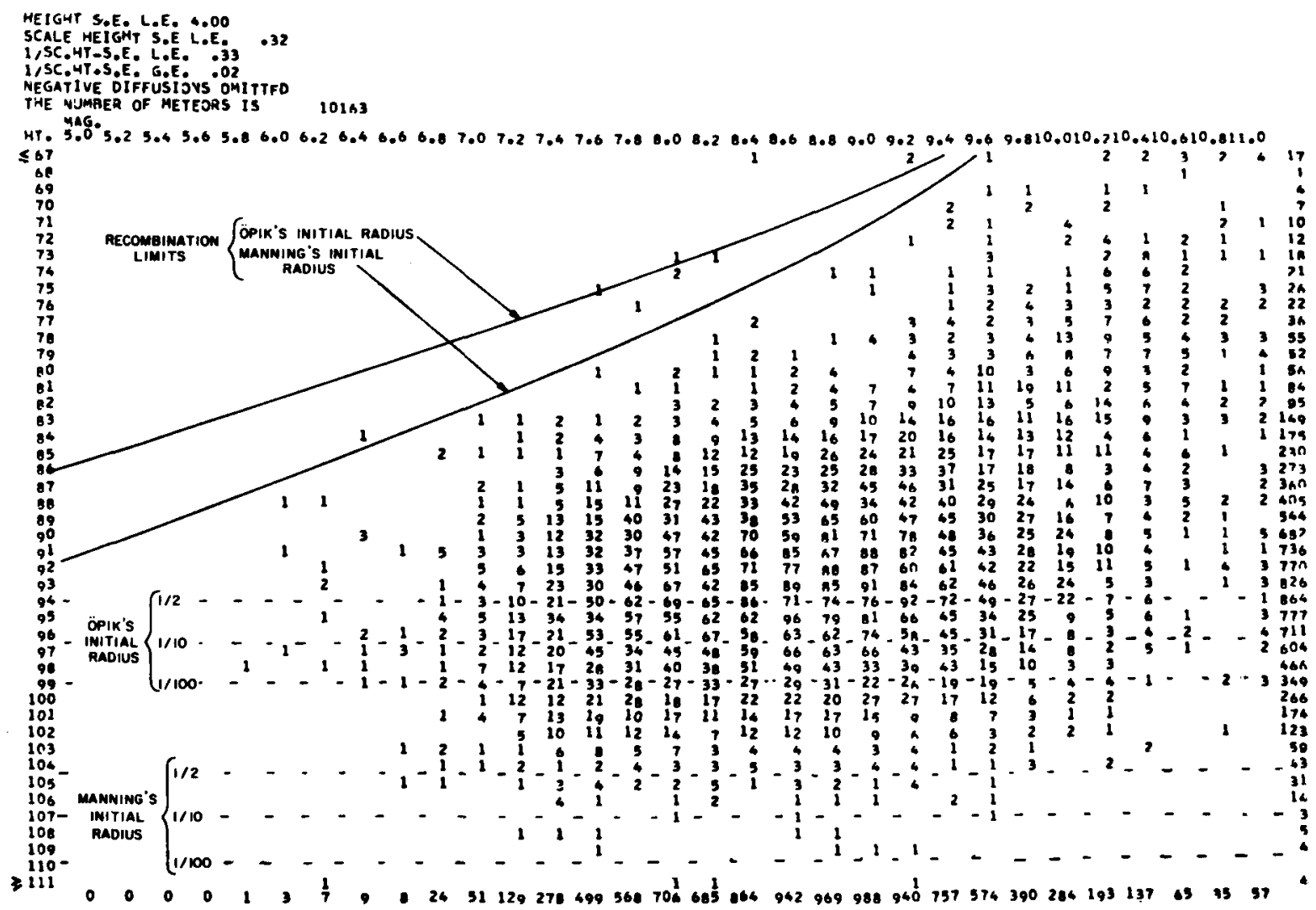

Figure 2.-Diffusion heights at maximum ionization of 10163 meteors, as a function of radar magnitude at maximum ionization. The recombination limits are from equation (8). The initial radius limit is shown for received voltage attenuations of $1 / 2,1 / 10$, and $1 / 100$ (corresponding approximately to $1 / 2,1 / 10$, and $1 / 100$ in number of meteors observed). Considering that the standard errors of these heights are $4 \mathrm{~km}$ or less, these are good fits to the recombination limit and initial radius limit using Manning's initial radius.

effects that depend directly or inversely on atmospheric density, like diffusion, so that density changes with time do not smear the diagram.

Our second observation of recombination is in the lower bound to heights in figure 2. Figure 2 shows curves of $M_{1 \mathrm{im}}=35-2.5 \log _{10} q_{1 \mathrm{im}}$, using Manning's and Oopik's values of $r_{0}$ (exact values from Southworth, 1962) and $t=0.03 s$ (an average time for the center of the measured part of the Fresnel pattern, for different velocities and lengths of Fresnel pattern). The upper bound (eq. (10)) on observable radar magnitudes is well confirmed, as is Manning's initial radius. Öpik's initial radius is clearly disproved (for our magnitude range) by the observation of many meteors far above his ceiling in figure 2 . The actual upper bound to our observed heights is caused by diffusion, which cuts off our observations at lower heights than initial radius.

Attachment of electrons to neutral atoms or molecules has been proposed (Davis, Greenhow, and Hall, 1959) to explain an observed lower bound on radar heights of bright photographic meteors. Attachment cannot explain the lower bound to height in figure 2 , because attachment to atmospheric atoms or molecules would give a lower bound independent of magnitude, and atmospheric species vastly outnumber meteor atoms or molecules for these meteors. On the other hand, recombination seems to be an adequate explanation for Davis, Greenhow, and Hall's observations. The importance of attachment should be reconsidered with recombination taken into account. 
Our third observation of recombination appears in figure 3. This is similar to figure 2, except that more stringent limits have been placed on the scale height and on the standard error of the height of maximum. The relative distribution in figure 3 is essentially similar to that in figure 2 except that a band of meteors just above the recombination limit has been eliminated. This band represents the meteors where recombination is large enough to disturb the consistency between diffusion heights at different stations on the same meteor.

\section{FRAGMENTATION}

If the meteoroid is not a single body but has broken into fragments, the composite Fresnel pattern of the group can be constructed as the sum of individual Fresnel patterns. Differences in fragment size will cause differences in deceleration of the various fragments and will spread them along their common trajectory. In the composite Fresnel pattern of several or more fragments, this corresponds to smoothing out the later oscillations; and in the ionization curves constructed from observations and from single-body theory, this corresponds to progressively fainter magnitudes for the later Fresnel zones. This effect is obvious in figure 1 and in a large proportion of our meteors. It corresponds to fragment spreads of the order of 50 to $200 \mathrm{~m}$ along the trajectory; smaller fragment separations are below our resolution. A more complete study of fragmentation will be made at a later date.

\section{SYSTEMATIC ERRORS CAUSED BY RECOMBINATION}

Greenhow and Hall (1960) have made the most careful attempt, to date, to observe atmospheric density changes from study of meteors. They

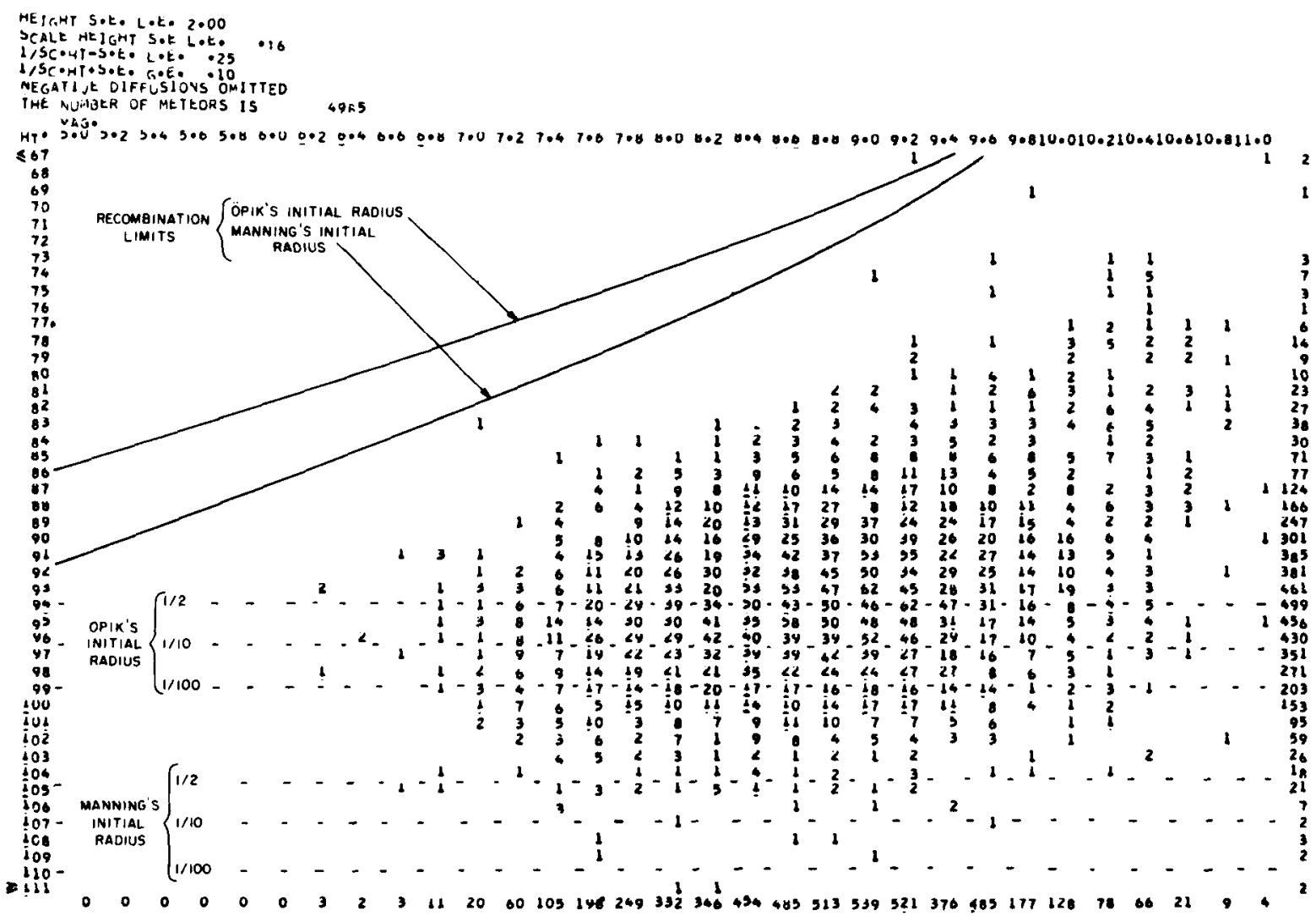

Figure 3.-Diffusion heights at maximum ionization of 4985 meteors as a function of radar magnitude at maximum ionization. These meteors are a subset of the meteors in figure 2, with standard errors in height of $2 \mathrm{~km}$ or less and with more closely restricted scale heights. 
measured diffusion from radar meteors as functions of height ( 85 to $118 \mathrm{~km}$ ) and time. Their rate of observation and lowest observed height show that they were observing magnitudes near the left edge of figure 2. It is not possible to correct their published observations for recombination; indeed, we could not correct more than a few if we had their original data. However, we can recognize the qualitative effects of recombination on their measures.

Three of Greenhow and Hall's principal results are out of harmony with other measures of the atmosphere: (1) the mean scale height was $9 \mathrm{~km}$; (2) the density at their mean height of $96 \mathrm{~km}$ varied regularly through the day, with a minimum near 0600 hours and a maximum near 1800; and (3) the scale height varied regularly through the day, with a minimum near 0600 and a maximum near 1800. A priori, it is suspicious that these variations should be in phase with the diurnal variation of meteor velocities, where high velocities predominate in the morning and low velocities in the evening. In fact, all three effects are readily explained in terms of recombination: (1) their mean scale height is too high because their lower meteors were much affected by recombination and their higher meteors hardly at all; (2) their density at $96 \mathrm{~km}$ varied because the faster meteors observed in the morning were observed sooner after the column was formed, so that the measured diffusion contained more recombination than the slow meteors in the evening at the same height; and (3) their scale height varied because the fast meteors in the morning were higher, so that the slope of the overall sample was less perturbed by recombination, while the evening sample consisted mostly of meteors with appreciable recombination. Figure 4 gives a schematic representation of these effects.

\section{SELECTION EFFECTS}

Recombination causes a quite unexpected selection effect against bright, low meteors. Since these are predominantly slow meteors, all existing statistics on velocity distributions of radar meteors have been significantly biased. The unrecorded meteors are mostly in direct orbits near the ecliptic plane, and it will also be necessary to revise present calculations on meteor space density

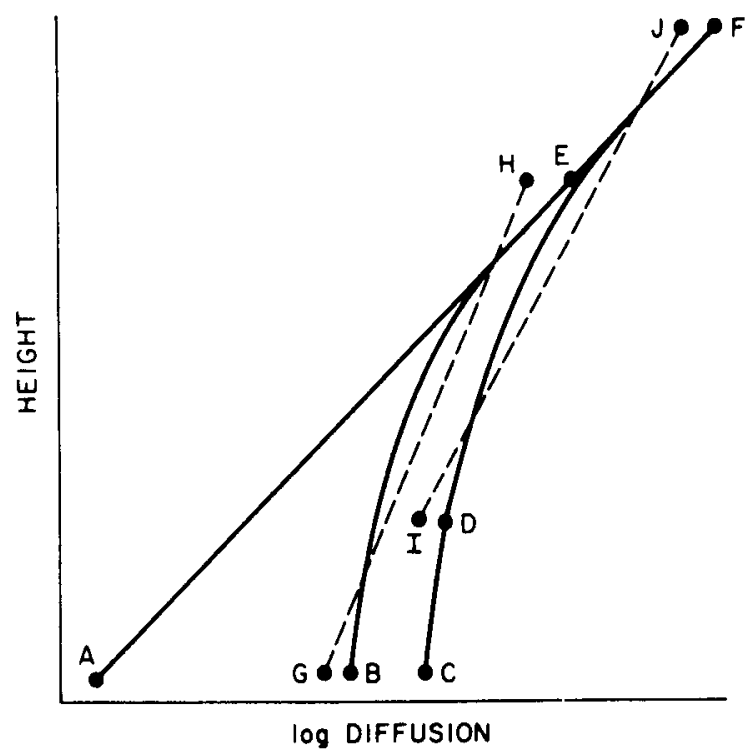

Figure 4.-Schematic explanation of Greenhow and Hall's results: $A F$-true diffusion; $B F$-diffusion plus recombination in the evening; $C F$-diffusion plus recombination in the morning; $G H$-linear fit to evening sample $B E ; I J$-linear fit to morning sample $D F$.

(Southworth, 1967). With the Havana equipment, we will usually be able to recognize meteors that have been affected by recombination; but we will often be unable to correct for it, because most of the recombination occurs before we observe the meteor. Simultaneous observations with the image orthicon and the radar are the best way to determine how many meteors are missed or undervalued because of recombination.

\section{IONIZING PROBABILITY}

Theoretical treatment of ionizing probability in meteors suffers from grave difficulties; the energy levels are too high or too low for present techniques, the physical processes are little known, and the meteor composition is not known. In practice, the ionizing probability has been evaluated by comparing radar observations with photographic or visual observations. Verniani and Hawkins (1964) wrote the latest and most thorough discussion, combining simultaneous radar-visual observations (Millman and McKinley, 1956) with the handful of available radar-photographic observations (Davis and Hall, 1963). They found a large scatter in the data 
but agreed on

$$
\beta=10^{-20} v^{-4}
$$

for the probability that an ablated meteor atom would be ionized (assuming that only meteor atoms are ionized in the first instance), where velocity $v$ is in centimeters per second.

Unfortunately, recombination essentially vitiates all existing determinations of ionizing probability made by use of visual or photographic meteors. If we extend the recombination limit (using Manning's initial radius) in figure 1 to greater heights, we reach magnitude +4 at $100 \mathrm{~km}$ and magnitude +2 at $110 \mathrm{~km}$, implying that virtually all visual and photographic meteors are affected by recombination to some extent. Of course, this extension can hardly be quite correct; the initial radius probably depends on magnitude, and the early history of the ionized column doubtless depends on the ratio of meteoric atoms and molecules to atmospheric atoms and molecules. Recombination depends very strongly on both these factors. If, however, we guess that Öpik's initial radius may be a better approximation for brighter meteors, we still find that most visual and photographic meteors experience recombination. Further, if we assume that we know the initial radius and the physical processes, a rediscussion of the observations considering recombination is still unpromising because the uncertainties in magnitude, and in radar and optical height, make it difficult even to recognize unrecombined meteors, and correction for recombination is impossible. The most hopeful view is that radar-visual observations of faint high meteors may be little affected by recombination. However, we retain uncertainties in the initial radius and in the early history of the ion column; the former uncertainty is an additional difficulty for analysis of radar echoes from high meteors.

The only observational treatment of ionizing probability made without use of optical observations was done by Evans and Hall (1955). They conclude, using the slope of the height-velocity curve and a theoretical height-magnitude relation, that ionizing probability is independent of velocity. This conclusion is invalidated by the observed independence of height and magnitude, as seen in figure 2.

If we assume that recombination is the only significant effect omitted from past determina- tions of ionizing probability, it appears that equation (18) must represent a lower bound. However, equation (18) might not be a poor approximation if there were a self-selection effect whereby recombining meteors tended to give radar echoes below equipment-sensitivity limits and thus tended to be omitted from statistics of simultaneous observations. In view of the other uncertainties dependent on magnitude (initial electron radius, fragment spread, early history of the column), we can conclude very little from the data analyzed so far.

Simultaneous radar and image-orthicon observations are the only resource now available to us for determining ionizing probability in our magnitude range and for finding masses for our radar meteors.

\section{CONCLUSIONS}

We have observed recombination in the ionized columns generated by faint radar meteors (radar magnitudes 6 to 9 ) as: (1) a rapid loss of returned signal in the first few milliseconds after formation of the column, before the slower decay caused by diffusion; (2) an apparent absence of bright, low meteors; and (3) anomalies in apparent diffusion rates. Recombination at rates characteristic of dissociative recombination of ionized atmospheric molecules $\mathrm{N}_{2}{ }^{+}$and $\mathrm{O}_{2}{ }^{+}$is completely consistent with the observations. Since no other likely constituent of the atmosphere or of the meteoroid has a fast enough recombination rate, it appears either that the molecules are ionized in the initial formation of the ionized column or that there is rapid charge exchange.

Recombination is a sufficient cause for the differences between Greenhow and Hall's (1960) diffusion measures and other atmospheric studies.

Manning's (1958) estimate of the initial radius of meteor columns is much more nearly correct than Opik's (1955) for radar magnitudes 6 to 11 .

Bright, low, slow meteors have been missed in radar observations, so that existing statistics are significantly biased. All past determinations of the ionizing probability based on bright meteor observations are invalid.

\section{ACKNOWLEDGMENTS}

This work was supported in part by contract NSR 09-015-033 from the National Aeronautics and Space Administration. 


\section{REFERENCES}

Davis, J., Greenhow, J. S., and Hall, J. E., 1959. The effect of attachment on radio echo observations of meteors, Proc. Roy. Soc. London, A, 253, 130-139.

Davis, J., And Hall, J. E., 1963. Meteor luminosity and ionization, Proc. Roy. Soc. London, A, $271,120-128$.

Evans, G. C., ANd Hall, J. E., 1955. Meteor ionizing and luminous efficiencies, in Meteors, edited by T. R. Kaiser, Pergamon Press, New York, 18-22.

Greenhow, J. S., AND Hall, J. E., 1960. Diurnal variations of density and scale height in the upper atmosphere, J. Atmos. Terr. Phys., 18, 203-214.

Greenhow, J. S., AND Neufeld, E. L., 1955. The diffusion of ionized meteor trails in the upper atmosphere, J. Atmos. Terr. Phys., 6, 133-140.

KAISER, T. R., 1955. The interpretation of radio echoes from meteor trails, in Meteors, edited by T. R. Kaiser, Pergamon Press, New York, 55-64.

Manning, L. A., 1958. The initial radius of meteoritic ionization trails, J. Geophys. Res., 63, 181-196.

McKrnLeY, D. W. R., 1961. Meteor science and engineering, McGraw-Hill Book Co., New York, $309 \mathrm{pp}$.

Mrhr, T. J., AND Biondr, M. A., 1969. Electron temperature dependence of recombination of $\mathrm{O}_{2}{ }^{+}$and $\mathrm{N}_{2}{ }^{+}$ions with electrons, Phys. Rev., 181, 264-271.

Millman, P. M., AND McKinley, D. W. R., 1956. Meteor echo durations and visual magnitudes, Can. J. Phys., 34, 50-61.

ÖPIK, E. J., 1955. Meteors and the upper atmosphere, Irish Astron. J., 3, 165-181.

OPIK, E. J., 1958. Physics of meteor fight in the atmosphere, Interscience Publishers, New York, $174 \mathrm{pp}$.

Southworth, R. B., 1962. Theoretical Fresnel patterns of radio meteors, Harvard-Smithson. Radio Meteor Proj. Res. Rept. No. 14, 60 pp.

- 1967. Space density of radio meteors, in The Zodiacal Light and the Interplanetary Medium, edited by J. L. Weinberg, NASA SP-150, Supt. of Documents, U.S. Govt. Printing Office, Washington, 179-188; also in Smithson. Astrophys. Obs. Spec. Rept. No. 239, 75-98.

Verniani, Franco, and Hawkins, G. S., 1964. On the ionizing efficiency of meteors, Astrophys. $J ., 140,1590-1600$. 